\title{
BIOMATERIALS
}

\section{Designing new biointerfaces with silicon}

Jiang, Y. et al. Nat. Biomed. Eng. https://doi.org/10.1038/s41551-018-0230-1 (2018).

Silicon may be best known as the stuff of cell phones, computers, and the eponymous valley in California, but it's also been making its way into biological applications. The semiconductive metalloid element is uniquely suited among others on the periodic table for use as a biomaterial, notes materials scientist Bozhi Tian. There are two advantages for building implantable devices with it. "It is, in my perspective, the most biocompatible semiconductor we know so far, and it's also biodegradable," he says.

Tian started working with silicon about a decade ago. As a graduate student in Charles Lieber's lab at Harvard, he helped develop silicon-based nanowires that could record electrical activity in samples of heart cells (Science 329, 830-834; 2010). Now with his own lab at the University of Chicago, Tian wants to create devices that can both sense and stimulate. His latest paper, led by his $\mathrm{PhD}$ student Yuanwen Jiang, presents the proof-of-concept for silicon biointerfaces that can be used at three different scales: organelles, tissues, and organs in vivo. The key was a rational design, and a little light.

Rational design is a decision-based design theory frequently used in chemical biology and bioengineering to build new materials with a particular function. Tian's team knew what they wanted to do-modulate and record activity from organelles to organs-but to make the devices work as intended they first needed to understand the physical properties of the different environments and how silicon behaved within them. Silicon is an effective energy transducer, Tian explains, with photonic and optoelectronic properties that allow it to convert a light source into biologically usable energy through three different physical processes: thermal (via heat), capacitive (via transient electricity), and Faradaic (via reduction/ oxidation reactions). Figuring out which was the most efficient process for each scale was the most challenging part of the study, Tian says. "It became rationally designed, but initially it was like a random walk." But with time, testing, and lots of screening of different
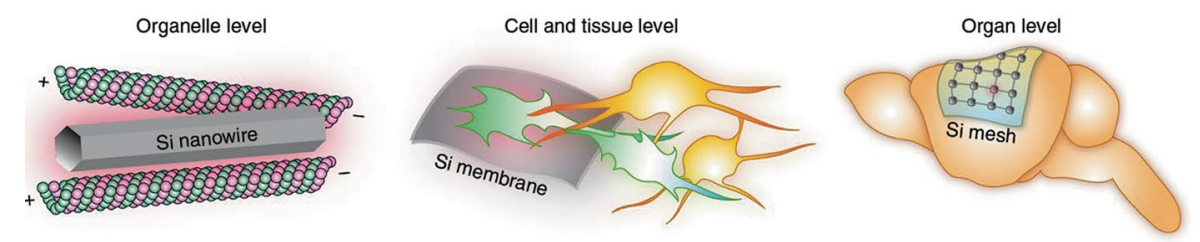

Silicon across scales. Reprinted with permission from Jiang et al. (2018), Springer Nature

devices, the best approaches for each application emerged.

At the intracellular scale of organelles, the team created silicon nanowires that could enter a mammalian cell via phagocytosis. Because little is yet known about the chemical or physical properties inside cells, they determined that transient heating would be best. With their nanowires, they demonstrated that they could create transient pores that released calcium ions into the cytosol of a cell, and also break apart cytoskeleton filaments. A bit more energy is needed to operate at larger scales, but tissue can be damaged by too much heat. To operate in small tissues, they created a silicon membrane controlled by capacitive processes, using light input to cause a spike of electricity. In the paper, they used their membrane to activate presynaptic neurons in a sample of mouse neocortex.

However, electricity alone wouldn't do the trick in larger tissues and organs. For biological control at this largest scale, the team came up with a gold-coated silicon mesh that could transfer light energy through both capacitive and Faradaic processes (chemical changes prompted by the latter can diffuse deeper into tissues). They tested their mesh in vivo, surgically laying it on the brain surface of C57BL/6J mice. Depending on where on the mesh they applied their light source (a laser with about the intensity of an LED light), they could enhance brain activity in the somatosensory cortex or control the motion of different limbs through the motor cortex.

Charles Lieber, who was not involved with the current study, was excited by the advances shown in the paper. "They introduced an important conceptual framework to enable biology-guided design of interfaces and devices with biological systems," he commented via email. "Significantly, they also demonstrate an application of this work by effecting simple rodent behaviors with optically controlled freestanding (i.e., interconnect-free) Si mesh interfaced to the cerebral cortex."

Such neuromodulation isn't newelectrical stimulation and optogenetics have been common in vivo techniques for some time now. But Tian thinks silicon interfaces will be a useful improvement for other researchers, particularly those working with larger species like nonhuman primates or one day even humans in clinical settings where the current approaches have drawbacks. Electrical stimulation is efficient at influencing neuronal activity, but fixed metal electrodes and wires can cause inflammation over time and can't be easily moved or removed without additional surgeries, while optogenetic techniques require gene editing to target specific regions of the brain. "Our approach actually combines advantages of the existing two methods," he says: non-genetic, targeted stimulation via light.

Though the depth was shallow and the animals were head-fixed for this proof-of-concept work, Tian is currently trying to improve the interfaces to function in deeper tissue and in freely moving animals, now that he and his lab understand the underlying physical properties involved. On to a "silicon standard" of the future?

Ellen P. Neff

Published online: 25 June 2018

https://doi.org/10.1038/s41684-018-0096-6 